\title{
Ear/Eye Drops, Suspension Dosage Form
}

National Cancer Institute

\section{Source}

National Cancer Institute. Ear/Eye Drops, Suspension Dosage Form. NCI Thesaurus.

Code C149468.

Liquid sterile preparation consisting of a suspension intended for use as ear drops or eye drops. 